\title{
Tuning the Optoelectronic Properties of Cross Conjugated Small Molecules Using Benzodithiophene As a Core Unit With Favorable Photovoltaic Parameters
}

\section{Sana Majeed}

UAF: University of Agriculture Faisalabad

Shafiq Ur Rehman

University of Agriculture Faisalabad

Anjum Zia

University of agriculture, faisalabad

Javed lqbal ( $\sim$ javedkhattak79@gmail.com)

University of Agriculture Faisalabad https://orcid.org/0000-0003-0598-8401

\section{Research Article}

Keywords: Benzo [1,2-b:4,5-b囚] (BDT), cross conjugated molecules, organic photovoltaic cells

Posted Date: October 22nd, 2021

DOI: https://doi.org/10.21203/rs.3.rs-965525/v1

License: (c) (1) This work is licensed under a Creative Commons Attribution 4.0 International License.

Read Full License 


\section{Abstract}

In a recent study, cross conjugated molecules (BDT-C1 to BDT-C6) based on Benzo [1,2-b:4,5-b囚] (BDT) as core units linked with different acceptor moieties are designed for encouraging photovoltaic applications. The optoelectronic study has been conducted by density functional theory (DFT) at B3LYP 6-31G (d, p) basis set combination by equating them with recently reported cross conjugated reference (BDT-CR) molecule and to study basic parameters such as frontier molecular orbital, density of states, reorganization energy, maximum absorption, dipole moment, transition density matrix (TDM) and opencircuit voltage $\left(\mathrm{V}_{\mathrm{OC}}\right)$. Six new cross conjugated molecules (BDT-C1 to BDT-C6) with modified acceptor moieties are designed to evaluate their photophysical behavior in photovoltaic cells and the optoelectronic analysis of designed molecules indicates that among all cross conjugated molecules, BDTC3 molecule exhibited the lowest bandgap value $(1.83 \mathrm{eV})$ and broad absorption (747 nm) spectrum in dichloromethane (DCM) due to extended conjugation in molecule than BDT-CR. TDM results reveal the easy dissociation of exciton due to the transfer of electron density in a diagonal direction from donor to acceptor moieties. The lowest value of electron mobility $(0.0030 \mathrm{eV})$ and hole mobility $(0.0027 \mathrm{eV})$ of BDT-C4 indicates its excellent electron and hole transfer behavior. The newly architecture molecule BDTC5 displayed the highest $\mathrm{V}_{\mathrm{OC}}$ value of $1.75 \mathrm{eV}$ concerning $\mathrm{PC}_{61} \mathrm{BM}$. All above-mentioned outcomes reflects that our newly architecture cross conjugated molecules are suitable applicants for photovoltaic cells and can exhibit wonderful results in the quest of power conversion efficiency (PCE).

\section{Highlights}

- Benzodithiophene based cross conjugated molecules (BDT-C1 to BDT-C6) has been designed for photovoltaic applications.

- The recent investigated molecules exhibited good optoelectronic properties.

- The designed donor molecules showed significant charge transfer ability.

\section{Introduction}

Balance, accessible and consistent supply of energy is the basic, crucial and inevitable need for the development of modern societies. The global energy demand is increasing with time throughout history due to the rapid increase of human population, modernization and urbanization. In the coming years, the global energy demand will reach its maximum height to meet the energy requirements of human life [1, 2]. So, scientists and researchers are looking to explore alternative energy sources and renewable energy sources (tidal, wind power, biomass, solar energy) are considered to be environment friendly, abundant and inexhaustible to cope with the rapidly increasing current global energy demand without any environmental degradation. Among all of these potential alternatives, solar energy is propitious and of great interest with fascinating features to overcome the energy deficit. Solar energy is the cleanest, safe and appealing source of energy and photovoltaic cells are of various types such as gallium-selenidebased photovoltaic cells, monocrystalline silicon-based solar cell, dye-sensitized photovoltaic cell and 
hybrid, solution-processed, perovskite and organic small molecules based solar cells. The silicon-based solar cell has remarkable efficiency but their use in energy conversion technology is limited due to many limitations such as high cost, rigid structure and complicated fabrication process which requires a high range of temperature and vacuum. While organic solar cells are the best alternative to silicon-based solar and their use in solar to energy conversion technologies is increasing day by day due to the use of small organic molecules. Organic solar cells gained more attention and have become to be an auspicious and potential technology because of their tremendous benefits including lightweight, environment friendly, low fabrication cost and mechanical stability [3]. PCE of $13 \%$ has been obtained for small moleculebased organic photovoltaic cells and many efforts have been exerted to attained further remarkable power conversion efficiency. Small molecule-based organic photovoltaic cells have many fascinating features in contrast to solution-processed polymer solar cells but still have some limitations like energy levels of donor molecules do not align with that of acceptor molecules, poor charge transport and film morphology which affect open-circuit voltage. To overcome the above-cited shortcomings, scientists and researchers have designed novel and efficient alternating small molecule donor materials [4-13]. Traditionally, fullerene-based acceptor molecules had been used to obtain the high PCE value. These frequent acceptor molecules allow efficient transport of charge and ultrafast charge separation but their optical absorption in the visible region is very poor and has highly complicated synthesis procedures as compared to small molecules and other polymers $[14,15]$. Recently, highly efficient non-fullerene acceptor (NFAs) molecules are of great consideration and have to gain more attention because of the tenability of their optical properties and strong absorption of electromagnetic radiations in the visible region $[16,17]$.

Organic photovoltaic cells are evaluated by photovoltaic parameters, optoelectronic properties and opposite architecture. Concerning morphology, OPVs are contrived in viable methods. The first approach is to construct the OPVs as single layer OSCs comprise of only one active organic layer that is sandwich between two respective electrodes named anode and cathode. But this type of cell accounted for low conversion and quantum efficiency. The second strategy encompasses the electron donor (D) and acceptor (A) layer to enhance the operational performance of OPVs. The third and most important development approach to enhance the efficiency of OPVs is bulk heterojunction or dispersed OSCs comprised of a blend of donor and acceptor materials. This effective strategy can overcome the worldwide power outage by boost up the operational efficiency of OPVs.

Recently, benzo [1,2-b:4,5囚]dithiophene (DBT) as a central fused core is of great interest due to its desirable quantum efficiency [18]. In photovoltaic cells, pi-conjugated organic semiconductors played a significant role in the ease of charge density transfer from $D$ to A moiety resulted in high operational efficiency of the photovoltaic cell. BDT has been used as donor material and has exhibited efficient and excellent photovoltaic properties. The blend of small molecule-based donor and acceptor moieties enhances the optoelectronic properties of solar cells due to different substitutions on the BDT core unit [19-22]

Herein, we did a computational study on different cross conjugated molecules BDT-C1 to BDT-C6 which possessed a fused core of BDT due to its peculiar behavior as it has symmetrical and planner structure, 
excellent charge transport properties and structural modification versatility [23]. In the current initiative, the reference BDT-CR and new cross conjugated molecules BDT-C1toBDT-C6 consists of BDT as a donor (core unit) linked with different acceptors moieties via thiophene (see Fig. 1). The introduction of thiophene in all molecules can facilitate the charge shifting from the core to the end-capped acceptor groups at the terminal by alleviating conjugation and boost up the donating capability of BDT. The calculated optical and electronic features of cross conjugated novel molecules were equated with BDT-CR molecule [24] and the outcomes of all investigated optoelectronic properties suggest that these newly designed cross conjugated molecules could be used as a promising avenue to enhance the efficiency of the organic photovoltaic cell.

\section{Computational Study}

Gaussian 09 software [25] was used for all types of calculations of novel architecture molecules in the present chemical study. The Gaussview program was employed to construct the 3-D structures of the reference BDT-CR and designed molecules BDT-C1 to BDT-C6 and also to visualize the output of these calculations. DFT which gives the most reliable results was implemented to evaluate the spectroscopic, electronic and electronic properties [26]. The geometry of cross conjugated BDT-CR molecule was optimized via four hybrid functionals named MPW1PW91[27], PBEPBE[28], B3LYP [29] and WB97XD using 6-31G $(d, p)$ basis set method. The solvent effect was investigated using the integral equation formalism polarizable continuum model (IEPCM) (a solvation modal) by employing DCM as solvent [30]. An important approach known as Time-dependent density functional theory (TD-DFT) [31]with a functional method of B3LYP and basis set of 6-31G $(\mathrm{d}, \mathrm{p})$ was employed in both gaseous and solvent medium for modeling electronic and excited-state energy calculations. The absorption spectra of reference and all examined cross conjugated molecules were plotted using origin 6.0 software [32]. The valid theoretical approach was selected by equating the calculated maximum absorption $\left(\lambda_{\max }\right)$ of the BDT-CR with that of experimental $\left(\lambda_{\max }\right)$ value. B3LYP was selected for geometry optimization and further calculations of all newly designed cross conjugated molecules due to the closest $\left(\lambda_{\max }\right)$ value of this

method to experimental reported $\lambda_{\max }$ value as compared to other functional methods. Therefore selected functional B3LYP/6-31G (d,p) approach was employed for DOS calculation by using PyMolyze 1.1 software [33] , TDM analysis by using Multiwfn 3.7 program [34], dipole moment and FMOs of reference BDT-CR and tailored molecules BDT-C1toBDT-C6. Reorganization energy of BDT-CR and BDT-C1toBDT-C6 are divided into external and internal reorganization energy $\left(\lambda_{\text {ext }}\right.$ and $\left.\lambda_{\text {int }}\right) . \lambda_{\text {ext }}$ concerns with the polarization effect in external environment while $\lambda_{\text {int }}$ concerns with variation in internal structure. Herein, only the internal reorganization effect is of our interest and we discount the external effect in the current study due to our small donor molecules with a low dielectric constant value. Reorganization energy due to electron mobility $(\lambda e)$ can be determined from the below-mentioned equation (1)

$$
\lambda_{e}=\left[E_{-}^{0}-E_{0}\right]+\left[E_{0}^{-}-E_{-}\right]
$$


$E_{0}^{-}$represents the neutral molecule energy of the optimized anion. $E_{0}$ represents the energy of a neutral optimized molecule $E_{0}^{-}$denotes the energy of anion of optimized neutral molecule and $E_{-}$denotes the energy of anion of optimized anion of neutral molecule. Likewise, reorganization energy due to hole mobility $\left(\lambda_{h}\right)$ can be evaluated from the below-mentioned equation (2)

$$
\lambda_{h}=\left[E_{+}^{0}-E_{0}\right]+\left[E_{0}^{+}-E_{+}\right]_{(2)}
$$

$E_{+}^{0}$ represents the neutral molecule energy of optimized cation. While $E_{+}^{0}$ and $\mathrm{E}_{+}$signify cationic energy of the optimized neutral molecule and cationic energy of optimized cation of neutral molecule respectively.

\section{Results And Discussion 3.1. Optimized geometry}

The geometry of BDT-CR molecule was optimized via four distinct types of functionals such as B3LYP, PBEPBE, MPW1MP91 and WB97XD at 6-31G $(d, p)$ level of theory to choose the best choice for novel architecture cross conjugated molecules. The BDT-CR cross conjugated molecule exhibited the $\lambda_{\max }$ values of 395, 554, 350 and $312 \mathrm{~nm}$ at B3LYP, PBEPBE, MPW1MP91 and WB97XD respectively. The above-mentioned values were sensibly equated with an experimentally reported $\lambda_{\max }$ value of $407 \mathrm{~nm}$ [35] and calculated $\lambda_{\max }$ of the BDT-CR molecule with B3LYP functional has close approximation with experimental value (see Fig. 2)so B3LYP was nominated as an appropriate method to intensify optoelectronic features and for further calculations of all investigated cross conjugated molecules. The optimized geometries of recently studied molecules BDT-C1 to BDT-C6 which are the derivatives of cross conjugated BDT-CR and have donor-spacer-acceptor-arrangement has been portrayed in Fig. 3. Structure tailoring of cross conjugated BDT-CR molecule has been executed by keeping the donor fragment fixed and substituting the thiophene bridge with six different linkers name as 2-ethylidenemalononitrile (BDTC1), (Z)-methyl 2-cyanobut-2-enoate (BDT-C2),(E)-2-(2-ethylidene-5,6-diflouro-3oxo-2,3-dihydro-1-H-inden-1ylidene)malononitrile(BDT-C3), benzo[c][1,2,5]thiadiazole (BDT-C4), 2-((E)-((E)-5-ethylidene-3-methyl-4oxothiazolidin-2-ylidene)methyl)malononitrile (BDT-C5), (E)-5,ethylidene-3methyl-2-thioxothiazolidin-4-one (BDT-C6). The ground state optimized configurations of BDT-CR and designed cross conjugated molecule (BDT-C1 to BDT-C6) with de-delocalization of pi-electrons can facilitate the charge mobility and can be recommended as good candidate for their applications in organic photovoltaic cells.

\subsection{Frontier Molecular orbital (FMOs) and Density of state (DOS) analysis}


The geometry of new architecture optimized molecules BDT-C1toBDT-C6 with B3LYP functional using 6$31 \mathrm{G}(\mathrm{d}, \mathrm{p})$ basis set level has been exposed in Fig. 3. All optimized molecules have cross conjugated configuration exhibited donor-spacer-acceptor arrangement. Electronic distribution pattern of FMOs: highest occupied and lowest unoccupied molecular orbital (HOMO and LUMO) influences the photoelectronic properties of photovoltaic cells. FMOs are helpful to divulge the charge transition character of all investigated molecules.

The FMOs electronic distribution pattern of BDT-CR and designed cross conjugated designed molecules BDT-C1 to BDT-C6 is presented in Fig. 4. The HOMO-LUMO bandgap $\left(\mathrm{E}_{\text {HOMO }}-\mathrm{E}_{\mathrm{LUMO}}\right)$ indicates the chemical reactivity of the substituted entities and molecules with small band gaps $\left(E_{g}\right)$ are characterized as soft molecules because undoubtedly they have strong chemical reactivity and show low stability. BDT-CR molecule has an experimental HOMO energy value of $-5.19 \mathrm{eV}$ and LUMO value of $-2.38 \mathrm{eV}$ with bandgap energy value of 2.81e V. In all devised cross conjugated designed molecule BDT-C1 to BDT-C6, charge carrier ability is enhanced via extended conjugation that is assisted by the electron delocalization within the molecular systems. Similarly, newly designed cross conjugated molecules BDT-C1 to BDT-C6 exhibited the HOMO values of $-5.51,-5.19,-5.41,-5.93,-5.50,-5.23 \mathrm{eV}$ respectively and the trend of HOMO energy value is counted as BDT-C4 > BDT-C2 > BDT-CR > BDT-C6 > BDT-C3 > BDT-C5 > BDT-C1. The LUMO energy values for designed molecules BDT-C1 to BDT-C6 is counted as $-3.39,-3.05,-3.57,-2.76,-3.34,-3.09$ $\mathrm{eV}$ respectively and trend of LUMO energy value is found as BDT-CR > BDT-C4 > BDT-C2 > BDT-C6 > BDTC5 > BDT-C1 > BDT-C3. The calculated energy gap value for BDT-C1 to BDT-C6 molecules was noted as 2.12, 2.13, 1.83, 2.16, 2.16, $2.13 \mathrm{eV}$ (Table. 1) with increasing trend of BDT-CR > BDT-C4 = BDT-C5 > BDTC1 $>$ BDT-C2 $=$ BDT-C6 $>$ BDT-C3.

Table 1. The HOMO-LUMO (eV) and band gap (eV) energy values for BDT-CR and BDT-C1 to-BDT-C6 molecules

\begin{tabular}{|lrrl|}
\hline Molecule & HOMO $(\mathrm{eV})$ & LUMO $(\mathrm{eV})$ & Band Gap (eV) \\
\hline BDT-CR & -5.22 & -2.46 & 2.76 \\
BDT-C1 & -5.51 & -3.39 & 2.12 \\
\hline BDT-C2 & -5.19 & -3.05 & 2.13 \\
\hline BDT-C3 & -5.41 & -3.57 & 1.83 \\
\hline BDT-C4 & -4.93 & -2.76 & 2.16 \\
\hline BDT-C5 & -5.50 & -3.34 & 2.16 \\
\hline BDT-C6 & -5.23 & -3.09 & 2.13 \\
\hline
\end{tabular}

Among all these molecules BDT-C3 cross conjugated molecule showed the lowest energy gap value because of electron-pulling nature of (E)-2-(2-ethylidene-5,6-diflouro-3-oxo-2,3-dihydro-1H-inden-1- 
ylidene)malononitrile moiety at the peripheral position. FMOs representation depicts that HOMO electron density is restricted on the donor fragment while LUMO electron density is concentrated on the bridge and acceptor fragment of molecules. DOS analysis was performed for all these molecules and the outcomes from this analysis verify the pieces of evidence demonstrated by FMOs results. In the present investigation, the pictographic display of DOS analysis represented by blue, green and red color lines for donor, bridge and acceptor fragments of devised molecules showed that HOMO of all the designed molecules are identical, demonstrating that these occupied molecular orbitals are arising from a central core of the designed molecule resulting in the transfer of electrons within energy domain during excitation process (see Fig. 5). The LUMO of all molecules BDT-C1 to BDT-C6 is almost the same with little variation indicating that lowest unoccupied orbital rising from acceptor unit of the molecules. Graphical representation of HOMO-LUMO and band gap values for BDT-CR and BDT-C1 to BDT-C6 is exposed in Fig. 6.

\subsection{Optical properties}

Absorption properties are explored to envisage the working of photovoltaic cells. The spectra of BDT-CR were computed in the gaseous and solvent medium at four distinct DFT methods as shown in Fig. 7. For computing the electronic transition of BDT-CR and designed cross conjugated molecules (BDT-C1 to BDTC6), absorption spectrum was recorded using B3LYP/6-31G (d, p) basis set combination in gas and DCM medium (Fig. 8). The experimental value of maximum absorption for reference BDT-CR molecule in both gas and DCM was found as $391 \mathrm{~nm}$ and $489 \mathrm{~nm}$ respectively. The recorded spectrum in the UV-visible region with an intense peak of design cross conjugated molecules seems to be a good approximation to show good photovoltaic properties in organic photovoltaic cells. The Calculated wavelength $\left(\lambda_{\max }\right)$, oscillator strength $\left(f_{o}\right)$, experimental wavelength $\left(\lambda_{\max }\right)$ and excitation energy $(\Delta \mathrm{E})$ parameter for BDT-CR and all investigated molecules (BDT-C1 to BDT-C6) with B3LYP/6-31G $(\mathrm{d}, \mathrm{p})$ basis set combination are observed in both (gaseous and DCM ) phases for photovoltaic performance (Tables 2 and 3). The BDT-CR and newly architecture cross conjugated molecules BDT-C1 to BDT-C6 showed the $\lambda_{\max }$ at 391, 660, 659, $746,614,610$ and $650 \mathrm{~nm}$ in a gaseous phase. The presence of DCM shifted the absorption towards redshift with broader peaks as compared to the gas phase due to stabilized delocalized electrons.

The $\lambda_{\max }$ in the presence of a solvent for the reference BDT-CR and designed molecules BDT-C1 to BDT-C6 was reported as $489,665,664,748,625,633$ and $649 \mathrm{~nm}$ respectively. So it was noted that solvent has a great effect to shift the absorption towards a longer wavelength. The order of $\lambda_{\max }$ in the presence of solvent among tailored molecules was noted as BDT-C3 > BDT-C1 > BDT-C2 > BDT-C6 > BDT-C5 > BDT-C4 $>$ BDT-CR. Exceptionally, BDT-C3 exhibited the highest red-shift value because of strong $\mathrm{F}$ atoms containing electron pulling acceptor moiety with extended conjugation. The aforementioned discussion predicts that BDT-C3 highest absorption (in DCM and gaseous medium) and lowest bandgap value that profoundly makes it an effective candidate for solar cell applications. 
Table 2. Estimation of Excitation energy $(\Delta \mathrm{E})$, oscillator frequency $\left(f_{\mathrm{o}}\right)$ and percent configuration interaction for BDT-CR and designed cross conjugated molecule BDT-C1 to BDT-C6 in gaseous phase.

\begin{tabular}{|c|c|c|c|c|c|c|c|}
\hline Molecule & $\lambda_{\max }^{\text {cal }}$ & $\lambda_{\max }^{\exp }$ & $\begin{array}{l}\text { Electronic } \\
\text { Excitation }\end{array}$ & $\Delta \mathrm{E}$ & $f_{o}$ & Transition & \% C.I. \\
\hline BDTCR & 391 & 470 & $\mathrm{~S}_{0} \rightarrow \mathrm{S} 1$ & 3.16 & 0.34 & $\mathrm{H} \rightarrow \mathrm{L}$ & 69 \\
\hline BDT-C1 & 659 & 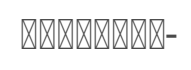 & - & 1.95 & 0.45 & - & 65 \\
\hline BDT-C2 & 659 & 囚- & - & 1.91 & 0.23 & - & 59 \\
\hline BDT-C3 & 746 & $\otimes-$ & - & 1.74 & 0.26 & - & 59 \\
\hline BDT-C4 & 614 & $\nabla-$ & - & 2.00 & 0.57 & - & 49 \\
\hline BDT-C5 & 610 & 凶- & - & 1.96 & 0.59 & - & 65 \\
\hline BDT-C6 & 649 & $\nabla-$ & - & 1.95 & 0.29 & - & 48 \\
\hline
\end{tabular}

Table 3. Estimation of first excitation energy $(\Delta \mathrm{E})$, oscillator frequency $\left(f_{\mathrm{o}}\right)$ and percent configuration interaction (C.I) for BDT-CR and designed cross conjugated molecules BDT-C1 to BDT-C6 in DCM solvent.

\begin{tabular}{|c|c|c|c|c|c|c|c|}
\hline Molecule & $\lambda_{\max }^{\text {cal }}$ & $\lambda_{\max }^{\exp }$ & $\begin{array}{l}\text { Electronic } \\
\text { Excitation }\end{array}$ & $\Delta \mathrm{E}$ & $f_{o}$ & Transition & \% C.I. \\
\hline BDT-CR & 489 & 407 & $S_{0} \rightarrow S_{1}$ & 2.53 & 0.54 & $\mathrm{H} \rightarrow \mathrm{L}$ & 69 \\
\hline BDT-C1 & 665 & - & - & 1.86 & 0.67 & - & 66 \\
\hline BDT-C2 & 664 & - & - & 1.87 & 0.45 & - & 58 \\
\hline BDT-C3 & 748 & - & - & 1.65 & 0.27 & - & 62 \\
\hline BDT-C4 & 625 & - & - & 1.98 & 1.21 & - & 59 \\
\hline BDT-C5 & 633 & - & - & 1.95 & 0.88 & - & 66 \\
\hline BDT-C6 & 649 & - & - & 1.91 & 0.45 & - & 55 \\
\hline
\end{tabular}


Transition density matrix (TDM) investigation of benzodithiophene-based cross conjugated small molecules was carried out for the estimation of transition nature and interaction of donor-acceptor groups during the excitation process. TDM describes the excitation process using a special map that displays electron-hole localization and transfer of charge density from donor to acceptor group [36, 37]. The selected B3LYP/ 6-31G (d, p) basis set combination was employed to evaluate the TDM analysis of BDT-CR and BDT-C1 to BDT-C6 cross conjugated molecules

Hydrogen atoms do not play an important role in transition so we neglected them in our recent studied molecules. TDM outcomes were evaluated by dividing the newly architecture cross conjugated molecules into three basic fragments as donor (benzodithiophene core), Spacer (thiophene) and different acceptors groups. Fig. 9 discloses the TDM display of BDT-CR and newly intended molecules (BDT-C1 to BDT-C6). The 2-D map indicates that in BDT-CR electron excitation is in the diagonal and off-diagonal character of donor and acceptor portion. The 2-D map of all tailored cross conjugated molecules presented outstanding diagonal charge transfer from BDT core to acceptor moiety via bridge representing smaller electron coupling in them and facilitates the easier exciton dissociation.

Another important parameter which helps to appraise the efficiency of organic photovoltaic cell is the binding energy and is a concrete tool helps to determine exciton dissociation potential and measures the Coulomb interaction of the hole and electron. The binding energy must be low for the dissociation of the exciton. Molecules with lower binding energy have a high potential of exciton dissociation during the transition to a higher energy state [37]. The binding energy of the BDT-CR and cross conjugated molecules BDT-C1 to BDT-C6 was calculated from the following equation (3) to evaluate the efficiency of an organic photovoltaic cell.

$E_{b}=E_{g}-E_{x}(3)$

Binding energy $\left(E_{b}\right)$ can be determined from the energy difference of HOMO and LOMO $\left(E_{g}\right)$ with first excitation energy $\left(E_{x}\right)$ for BDTR and all designed cross conjugated molecules. The binding energy of BDTCR and BDT-C1 to BDT-C6 has been computed in Table. 4. The trend of exciton binding energy for cross conjugated novel molecules is found as BDT-C6 $>$ BDT-C1 $=$ BDT-C2 $>$ BDT-CR $>$ BDT-C5 $>$ BDT-C3 $=$ BDTC4A lower value of binding energy resulted in higher current charge density $\left(\mathrm{J}_{\mathrm{SC}}\right)$ [38] and cross conjugated molecules BDT-C3 and BDT-C4 exhibited the lowest binding energy value than BDT-CR and other remaining molecules. So such molecules can be considered as well- suited for electronic excitation processes in photovoltaic cells.

Table 4. Excitation $\left.E_{(} \mathbf{s}_{1}-\mathbf{s}_{0}\right)(\mathrm{eV})$ and binding $E_{b}(e V)$ energies for BDT-CR and BDT-C1 to BDT-C6 in DCM. 


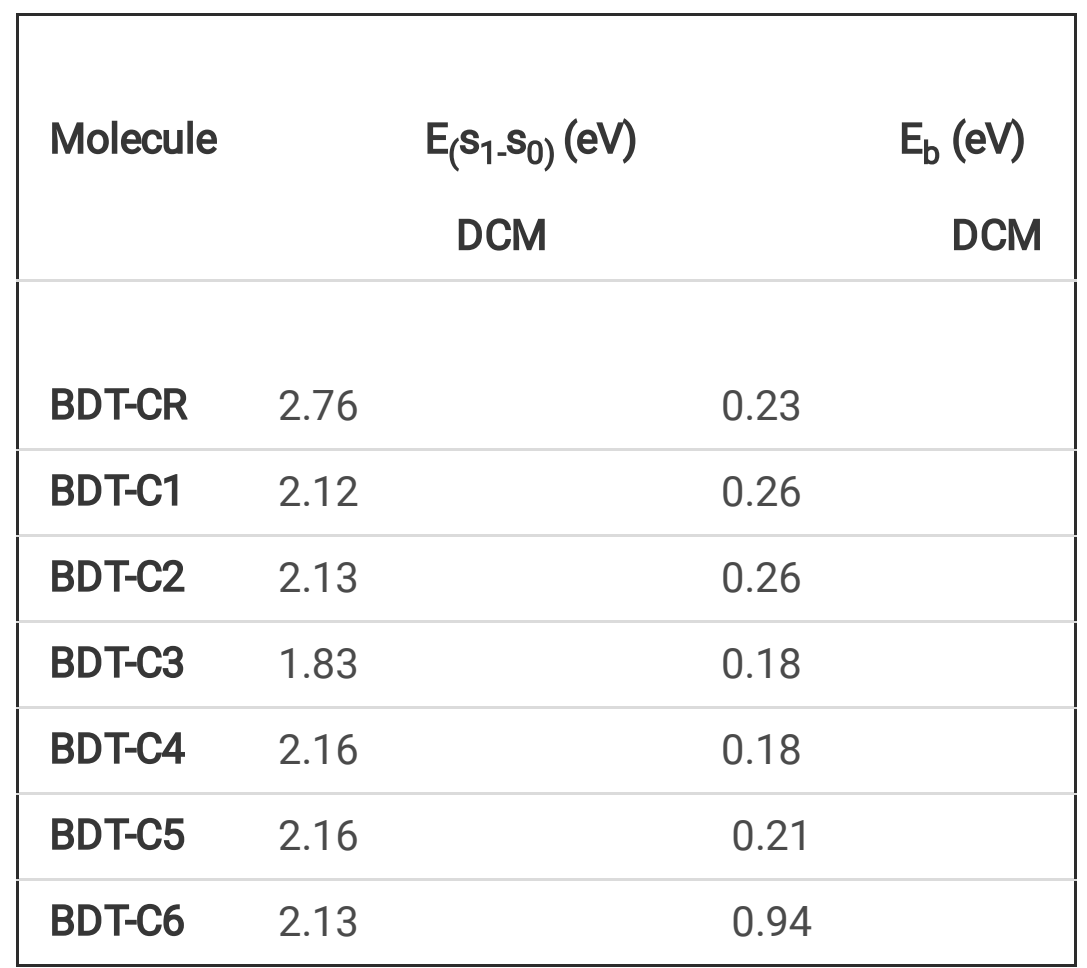

\subsection{Reorganization energy}

Reorganization energy (RE) is an auspicious and significant parameter to evaluate the performance of photovoltaic cells by hole and electron mobility. It has an inverse relation with the transfer of charge in the photovoltaic cell. Reorganization energy of material must be low in order to obtain high charge mobility. It is used to find out the total transfer of charge between donor and acceptor moiety. Different factors affect the reorganization energy of cells but it is primarily affected by the geometry of anion and cation [39].

Consequently, for the evaluation of electron and hole charge transport features for current studied cross conjugated molecules (BDT-C1 to BDT-C6), we have calculated RE values that were linked with different cationic and anionic geometries determined by applying Equations (1) and 2). Electron and hole reorganization energy value $\left(\lambda_{e}\right.$ and $\left.\lambda_{h}\right)$ for BDT-CR and all designed cross conjugated molecules BDT-C1 to BDT-C6 is tabulated in Table 5. Among all tailored molecules BDT-C4 exhibited the lowermost $\lambda_{\mathrm{e}}(0.0030 \mathrm{eV})$ and $\lambda_{\mathrm{h}}(0.0027 \mathrm{eV})$ values. Charge transport is increased as the cation and anion movement rates are reduced. So, as the result of the existence of a strong electron pulling fragment, BDTC4 has the highest cationic and anionic charge movement rate as well as lowest binding energy among all recently developed chromophores. As a result, the customized molecule might be considered a promising choice for building OSCs with improved charge transfer capabilities.

Table 5. Reorganization energies with electron and hole mobilties $\left(\lambda_{e}\right.$ and $\left.\lambda_{h}\right)$ for reference BDT-CR and designed cross conjugated molecules BDT-C1 to BDT-C6 at B3LYP method. 


\begin{tabular}{|c|c|c|}
\hline Molecule & $\lambda_{e}$ & $\lambda_{h}$ \\
\hline BDT-CR & 0.0147 & 0.0063 \\
\hline BDT-C1 & 0.0040 & 0.0068 \\
\hline BDT-C2 & 0.0181 & 0.0197 \\
\hline BDT-C3 & 0.0044 & 0.0147 \\
\hline BDT-C4 & 0.0030 & 0.0027 \\
\hline BDT-C5 & 0.0043 & 0.0031 \\
\hline BDT-C6 & 0.0043 & 0.0042 \\
\hline
\end{tabular}

\subsection{Dipole moment}

Dipole moment is the fundamental attribute in the performance of proficient organic photovoltaic cells. Dipole moment has an effect on molecular solubility in organic solvents [40]. The dipole moment will be taken into account more when a molecule has more polar groups and high solubility in the solvent and also has a great influence on the charge mobility of the molecule with a high charge transfer rate during excitation [41]. The dipole moment of designed cross conjugated donor molecules also affect the selfassembly of molecules in the fabricating film which facilitates the fabrication process in solar cell device due to anti-parallel arrangement of adjacent molecules and can ultimately improve the crystallinity and order in bulk heterojunction organic solar cell device. The $\lambda_{h}$ of molecules can be improved by increasing the strength of the dipole moment. When a molecule has a strong propensity to pack, boosting the dipole moment enhances its ability to self-assemble, resulting in a lengthy supramolecular chain that assists the mobility of charges by letting electrons and holes travel more easily [42].

The calculation of dipole moments for cross conjugated molecules BDT-C1 to BDT-C6 was also carried out at the same functional and has a direct correlation with the molecular solubility. The calculated dipole moment of entitled molecules is shown in Table 6. The increased value of dipole moment resulted in smooth morphology. The calculated dipole moments for BDT-CR were found as 6.18 and 7.12 in lower and higher energy states respectively. The designed molecules BDT-C1 to BDT-C6 have a ground state dipole moment value of $0.99,5.42,5.90,4.58,0.52,5.51$ Debye correspondingly. Similarly, all cross conjugated molecules have excited state dipole moments of $1.23,7.23,7.54,5.61,0.86,6.86$ Debye respectively. The values of dipole moment showed that all molecules have a greater value of dipole moment in a higher energy state (excited state) in contrast to the ground state The order of calculated excited state dipole moment of BDT-CR and BDT-C1 to BDT-C6 is found as BDT-C3 > BDT-C2 > BDT-CR > BDT-C6 > BDT-C4 > BDT-C1 > BDT-C5. 
Table. 6 Calculated value of Dipole moments for BDT-CR and BDT-C1-BDT-C6 at B3LYP/6-31 G (d,p)

\begin{tabular}{|c|c|c|c|}
\hline Molecule & $\mu_{g}(D)$ & $\mu_{e}(D)$ & $\mu_{e}-\mu_{g}(D)$ \\
\hline BDT-CR & 6.18 & 7.12 & 0.94 \\
\hline BDT-C1 & 0.99 & 1.23 & 0.24 \\
\hline BDT-C2 & 5.42 & 7.23 & 1.81 \\
\hline BDT-C3 & 5.90 & 7.54 & 1.64 \\
\hline BDT-C4 & 4.58 & 5.61 & 1.02 \\
\hline BDT-C5 & 0.52 & 0.86 & 0.33 \\
\hline BDT-C6 & 5.51 & 6.86 & 1.35 \\
\hline
\end{tabular}

The results reveal that BDT-C3 has a higher dipole moment value than other proposed molecules because of its better solubility and photovoltaic stability in DCM. So, it can be recommended as a preferred candidate to be employed in the fabrication of thin film. The graphical representation of dipole moments for BDT-CR and newly architecture molecules (BDT-C1 to BDT-C6) is shown in Fig. 10

\subsection{Open circuit Voltage $\left(\mathrm{V}_{\mathrm{OC}}\right)$}

$\mathrm{V}_{\mathrm{OC}}$ is another crucial factor to estimate the organic photovoltaic device performance. It is the maximum voltage across the cell when no current flow and cell work at an open circuit [43]. Our designed donor molecules BDT-C1 to BDT-C6 along with fullerene derivative PCBM as acceptor was used in ordered to evaluate the $\mathrm{V}_{\mathrm{OC}}$ values. It is the energy difference between the HOMO energy level of the design donor cross conjugated molecule and the $L U M O$ energy level of the $\mathrm{PC}_{61} \mathrm{BM}$ polymer used as acceptor material. $A$ high value of $V_{O C}$ depends on the greater difference between the bandgap energy of HOMO and LUMO by keeping the HOMO at a low energy level and LOMO at a high energy level [44-47]. The energy gap was calculated between HOMO of newly architecture cross conjugated donor molecules and LUMO of illustrious $\mathrm{PC}_{61} \mathrm{BM}$ acceptor material as shown in Fig. 11. $\mathrm{PC}_{61} \mathrm{BM}$ was employed as acceptor material with HOMO and LUMO values of -6.1 and $-3.7 \mathrm{eV}$ and optimized at selected B3LYP/6-31 G (d, p) basis set combination for quantum chemical exploration.

The following equation (4) can be used to calculate the $\mathrm{V}_{\mathrm{OC}}$ of newly designed cross conjugated molecules. 


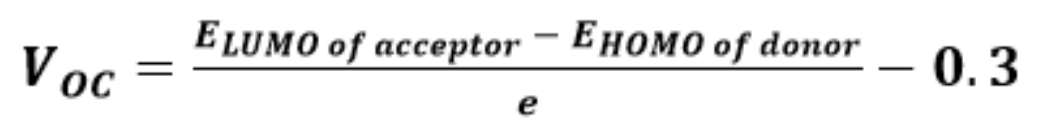

Herein, $\mathrm{E}$ is the energy gap of each investigated molecule and $\mathrm{e}$ is a charge on each molecule. The BDT$\mathrm{CR}$ and designed donor molecules (BDT-C1 to BDT-C6) showed the expected $\mathrm{V}_{\mathrm{OC}}$ value of 1.19, 1.69, 1.2, $1.55,1.15,1.75$ and $1.26 \mathrm{eV}$ respectively. All current studied designed cross conjugated molecules exhibited greater $\mathrm{V}_{\mathrm{OC}}$ due to their low-lying HOMO levels. BDT-C5 with heightened $\mathrm{V}_{\mathrm{OC}}$ value indicates effective electron transport behavior from HOMO (donor) to LUMO (acceptor). So, we deduce that BDT-C5: $\mathrm{PC}_{61} \mathrm{BM}$ composite could prove as an efficient active layer to boost up the working efficiency of organic photovoltaic cells.

\section{Conclusion}

In a recent quantum chemical investigation, cross conjugated molecules (BDT-C1 to BDT-C6) are designed to find their applications in an organic photovoltaic device. Different functionals (MPW1 MP91, PBEPBE, WB97XD and B3LYP) are employed to optimize the geometries of customized cross conjugated molecules. All parameters (optical and electrochemical) were determined at selected B3LYP/6-31 G $(d, p)$ basis set combination. All newly designed cross conjugated molecules consist of Benzo [1,2-b: 4,5-b冈] core unit linked with distinct acceptor moieties through thiophene. The photovoltaic parameters of intended cross conjugated molecules are evaluated by equating them with BDT-CR molecule. The $\lambda_{\max }$ of BDT-C1 to BDT-C6 in DCM solvent lies in the range of 489-748 nm exhibited red-shift behavior than BDTCR (407 nm). The absorption values in presence of solvent (DCM) are found to be high than the gaseous phase, indicates that DCM played an important role to move the absorption band towards red-shift. The bandgap values for newly planned molecules (BDT-C1 to BDT-C6) fluctuate between 1.83 and $2.16 \mathrm{eV}$ which are found to be less as compared to BDT-CR $(2.76 \mathrm{eV})$ facilitated the electrons transfer from HOMO to LUMO energy level. The maximum value of dipole moment (7.54 Debye) ofBDT-C3 showed that the probability of recombination of charges is less in it as compared to other molecules. Among all tailored molecules BDT-C4 has the highest electron and hole mobility value showed that this freshly designed molecule could have a plausibly greater chance of electron and hole transfer rate. 2-D map of TDM reveals that electron-density moves along diagonal direction allowing effective charge transfer from $D$ to A. Moreover, calculated $V_{0 C}$ values of BDT-C1-BDT-C6 concerning PC $_{61} B^{B M}$ suggest that they could transfer the maximum amount of current and can prove as credible candidates for designing efficient photovoltaic cells. Hence, our newly designed cross conjugated molecules would exhibit profound consequences to enhance the optoelectronic features and PCE of the organic photovoltaic cells.

\section{Declarations}

\section{Acknowledgment}

The authors acknowledge the financial and technical support from Punjab Bio-energy Institute (PBI), University of Agriculture, Faisalabad (UAF), and Pakistan. We also Thank Dr. Khurshid Ayub, Comsats 
university, Islamabad, Abbottabad campus, Pakistan for additional resources.

Funding: The authors would like to acknowledge the technical aid and financial support for this task from Punjab Bio-Energy Institute (PBI), University of Agriculture Faisalabad (UAF), Faisalabad-38000, Pakistan. We also thank Dr. Khurshid ayub, COMSATS University, Islamabad, Abbottabad campus, 22060, Pakistan for providing additional resources.

Conflicts of interest/Competing interests: All the authors declare no conflict of interest.

Availability of data and material: All data generated or analyzed during this study are included in this published article [and its supplementary information files].

Code availability: Not applicable

\section{Authors' contributions:}

1. Sana Majeed: The acquisition, analysis, or interpretation of data; drafted the work or revised it critically for important intellectual content.

2. Shafiq Ur Rehman: The acquisition, analysis, or interpretation of data; drafted the work or revised it critically for important intellectual content.

3. Anjum Zia: The acquisition, analysis, or interpretation of data; drafted the work or revised it critically

4. Javed Iqbal: Made substantial contributions to the conception or design of the work; the acquisition, revised it critically for important intellectual content; approved the version to be published; and agree to be accountable for all aspects of the work in ensuring that questions related to the accuracy or integrity of any part of the work are appropriately investigated and resolved.

\section{References}

1. Twidell, J. and T. Weir, Renewable energy resources. 2015: Routledge.

2. Asif, M. and T. Muneer, Energy supply, its demand and security issues for developed and emerging economies. Renewable and sustainable energy reviews, 2007. 11(7): p. 1388-1413.

3. Yu, J., Polymers, 2014, 6, 2473-2509;(d) L. Lu, T. Zheng, Q. Wu, AM Schneider, D. Zhao and L. Yu. Chem. Rev, 2015. 115: p. 12666-12731.

4. Lin, Y. and X. Zhan, Oligomer molecules for efficient organic photovoltaics. Accounts of chemical research, 2016. 49(2): p. 175-183.

5. Huang, J., et al., Highly efficient organic solar cells consisting of double bulk heterojunction layers. Advanced Materials, 2017. 29(19): p. 1606729. 
6. Kaltenbrunner, M., et al., Ultrathin and lightweight organic solar cells with high flexibility. Nature communications, 2012. 3(1): p. 1-7.

7. Krebs, F.C., et al., 25th anniversary article: rise to power-OPV-based solar parks. Advanced Materials, 2014. 26(1): p. 29-39.

8. Bruton, T., General trends about photovoltaics based on crystalline silicon. Solar Energy Materials and Solar Cells, 2002. 72(1-4): p. 3-10.

9. Khalil, A., et al. Review on organic solar cells. in 2016 13th International Multi-Conference on Systems, Signals \& Devices (SSD). 2016. IEEE.

10. Walker, B., C. Kim, and T.-Q. Nguyen, Small molecule solution-processed bulk heterojunction solar cells. Chemistry of Materials, 2011. 23(3): p. 470-482.

11. Ni, W., et al., Open-circuit voltage up to $1.07 \mathrm{~V}$ for solution processed small molecule based organic solar cells. Organic Electronics, 2014. 15(10): p. 2285-2294.

12. Dennler, G., M.C. Scharber, and C.J. Brabec, Polymer-fullerene bulk-heterojunction solar cells. Advanced materials, 2009. 21(13): p. 1323-1338.

13. Ans, M., et al., Designing Three-dimensional (3D) Non-Fullerene Small Molecule Acceptors with Efficient Photovoltaic Parameters. ChemistrySelect, 2018. 3(45): p. 12797-12804.

14. Nielsen, C.B., et al., Non-fullerene electron acceptors for use in organic solar cells. Accounts of chemical research, 2015. 48(11): p. 2803-2812.

15. Trukhanov, V. and D.Y. Paraschuk, Non-fullerene acceptors for organic solar cells. Polymer Science Series C, 2014. 56(1): p. 72-83.

16. Wen, S., et al., Benzodithiophene-based poly (aryleneethynylene) s: Synthesis, optical properties, and applications in organic solar cells. Journal of Polymer Science Part A: Polymer Chemistry, 2014. 52(2): p. 208-215.

17. Yan, C., et al., Non-fullerene acceptors for organic solar cells. Nature Reviews Materials, 2018. 3(3): p. 1-19.

18. Chen, C., et al., Application of benzodithiophene based A-D-A structured materials in efficient perovskite solar cells and organic solar cells. Nano Energy, 2016. 23: p. 40-49.

19. Gao, C., et al., Rational design on D-A conjugated P (BDT-DTBT) polymers for polymer solar cells. Polymer Chemistry, 2014. 5(18): p. 5200-5210.

20. Wang, Y.-L., Q.-S. Li, and Z.-S. Li, Novel benzodithiophene-based polymer acceptors for efficient organic solar cells. Physical Chemistry Chemical Physics, 2017. 19(34): p. 23444-23453. 
21. Ye, L., et al., Molecular design toward highly efficient photovoltaic polymers based on twodimensional conjugated benzodithiophene. Accounts of chemical research, 2014. 47(5): p. 1595-1603.

22. Xie, R., et al., Efficient Non-Fullerene Organic Solar Cells Based on a Wide-Bandgap Polymer Donor Containing an Alkylthiophenyl-Substituted Benzodithiophene Moiety. ChemPhysChem, 2019. 20(20): p. 2668-2673.

23. Zhou, J., et al., Solution-processed and high-performance organic solar cells using small molecules with a benzodithiophene unit. Journal of the American Chemical Society, 2013. 135(23): p. 8484-8487.

24. Lee, S.M., et al., Horizontal-, Vertical-, and Cross-Conjugated Small Molecules: Conjugated PathwayPerformance Correlations along Operation Mechanisms in Ternary Non-Fullerene Organic Solar Cells. Small, 2020. 16(5): p. 1905309.

25. Lange, A.W. and J.M. Herbert, A smooth, nonsingular, and faithful discretization scheme for polarizable continuum models: The switching/Gaussian approach. The Journal of chemical physics, 2010. 133(24): p. 244111.

26. Adamo, C., et al., Exploring excited states using time dependent density functional theory and densitybased indexes. Coordination Chemistry Reviews, 2015. 304: p. 166-178.

27. Adamo, C. and V. Barone, Exchange functionals with improved long-range behavior and adiabatic connection methods without adjustable parameters: The $m$ PW and $m$ PW1PW models. The Journal of chemical physics, 1998. 108(2): p. 664-675.

28. Yanai, T., D.P. Tew, and N.C. Handy, A new hybrid exchange-correlation functional using the Coulombattenuating method (CAM-B3LYP). Chemical physics letters, 2004. 393(1-3): p. 51-57.

29. Finley, J.P., Using the local density approximation and the LYP, BLYP and B3LYP functionals within reference-state one-particle density-matrix theory. Molecular Physics, 2004. 102(7): p. 627-639.

30. Mennucci, B., R. Cammi, and J. Tomasi, Excited states and solvatochromic shifts within a nonequilibrium solvation approach: A new formulation of the integral equation formalism method at the self-consistent field, configuration interaction, and multiconfiguration self-consistent field level. The Journal of chemical physics, 1998. 109(7): p. 2798-2807.

31. Ullrich, C.A. and Z.-h. Yang, A brief compendium of time-dependent density functional theory. Brazilian Journal of Physics, 2014. 44(1): p. 154-188.

32. Deschenes, L.A. and A. David A. Vanden BoutUniversity of Texas, Origin 6.0: Scientific Data Analysis and Graphing Software Origin Lab Corporation (formerly Microcal Software, Inc.). Web site: www. originlab. com. Commercial price: 595.Academicprice: 446. 2000, ACS Publications.

33. Tenderholt, A., PyMOlyze, Version 1.1. 2006, Stanford University, Stanford, CA. 
34. Lu, T. and F. Chen, Multiwfn: a multifunctional wavefunction analyzer. Journal of computational chemistry, 2012. 33(5): p. 580-592.

35. Baser-Kirazli, N., R.A. Lalancette, and F. Jäkle, Tuning the Donor- $\pi$-Acceptor Character of Arylborane-Arylamine Macrocycles. Organometallics, 2021. 40(4): p. 520-528.

36. Malmqvist, P.Å., Calculation of transition density matrices by nonunitary orbital transformations. International journal of quantum chemistry, 1986. 30(4): p. 479-494.

37. Ans, M., et al., Designing dithienothiophene (DTT)-based donor materials with efficient photovoltaic parameters for organic solar cells. Journal of molecular modeling, 2019. 25(8): p. 1-12.

38. Shehzad, R.A., et al., Designing of benzothiazole based non-fullerene acceptor (NFA) molecules for highly efficient organic solar cells. Computational and Theoretical Chemistry, 2020. 1181: p. 112833.

39. Bilal Ahmed Siddique, M., et al., Designing triphenylamine-configured donor materials with promising photovoltaic properties for highly efficient organic solar cells. ChemistrySelect, 2020. 5(25): p. 7358-7369.

40. Rasool, A., et al., Designing of benzodithiophene (BDT) based non-fullerene small molecules with favorable optoelectronic properties for proficient organic solar cells. Computational and Theoretical Chemistry, 2021. 1203: p. 113359.

41. Zahid, S., et al., Tuning the optoelectronic properties of triphenylamine (TPA) based small molecules by modifying central core for photovoltaic applications. Journal of Molecular Modeling, 2021. 27(9): p. 114.

42. Ans, M., et al., Designing of non-fullerene 3D star-shaped acceptors for organic solar cells. Journal of molecular modeling, 2019. 25(5): p. 1-12.

43. Tang, S. and J. Zhang, Design of donors with broad absorption regions and suitable frontier molecular orbitals to match typical acceptors via substitution on oligo (thienylenevinylene) toward solar cells. Journal of computational chemistry, 2012. 33(15): p. 1353-1363.

44. Bibi, S., et al., Effect of different topological structures (D- $\pi-D$ and $D-\pi-A-\pi-D)$ on the optoelectronic properties of benzo [2, 1-B: 3, 4-B] dithiophene based donor molecules toward organic solar cells. Solar Energy, 2019. 186: p. 311-322.

45. Xie, X., et al., Performance Regulation of Thieno [3, 2-b] benzothiophene $\pi$-Spacer-Based D- $\pi$-A Organic Dyes for Dye-Sensitized Solar Cell Applications: Insights From Computational Study. Frontiers in chemistry, 2019. 6: p. 676.

46. He, L.-J., et al., Fine-tuning $\pi$-spacer for high efficiency performance DSSC: a theoretical exploration with $D-\pi-A$ based organic dye. Dyes and Pigments, 2017. 141: p. 251-261. 
47. Pode, R., On the problem of open circuit voltage in metal phthalocyanine/C60 organic solar cells. Advanced Materials Letters, 2011. 2(1): p. 3-11.

\section{Figures}
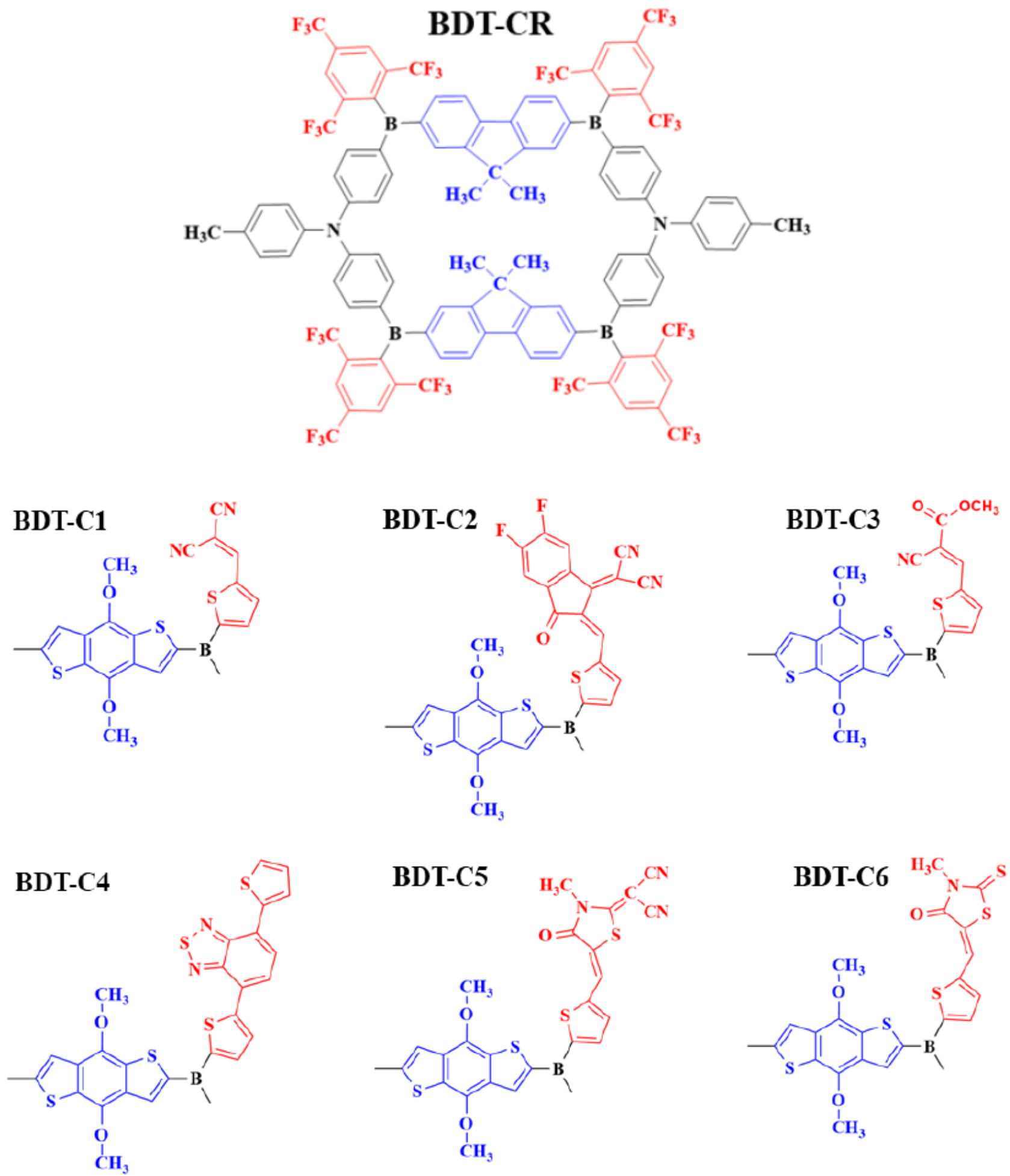

Figure 1 
Sketch map of molecular structures of BDT-CR and designed cross conjugated BDT-C1-BDT-C6 molecules.

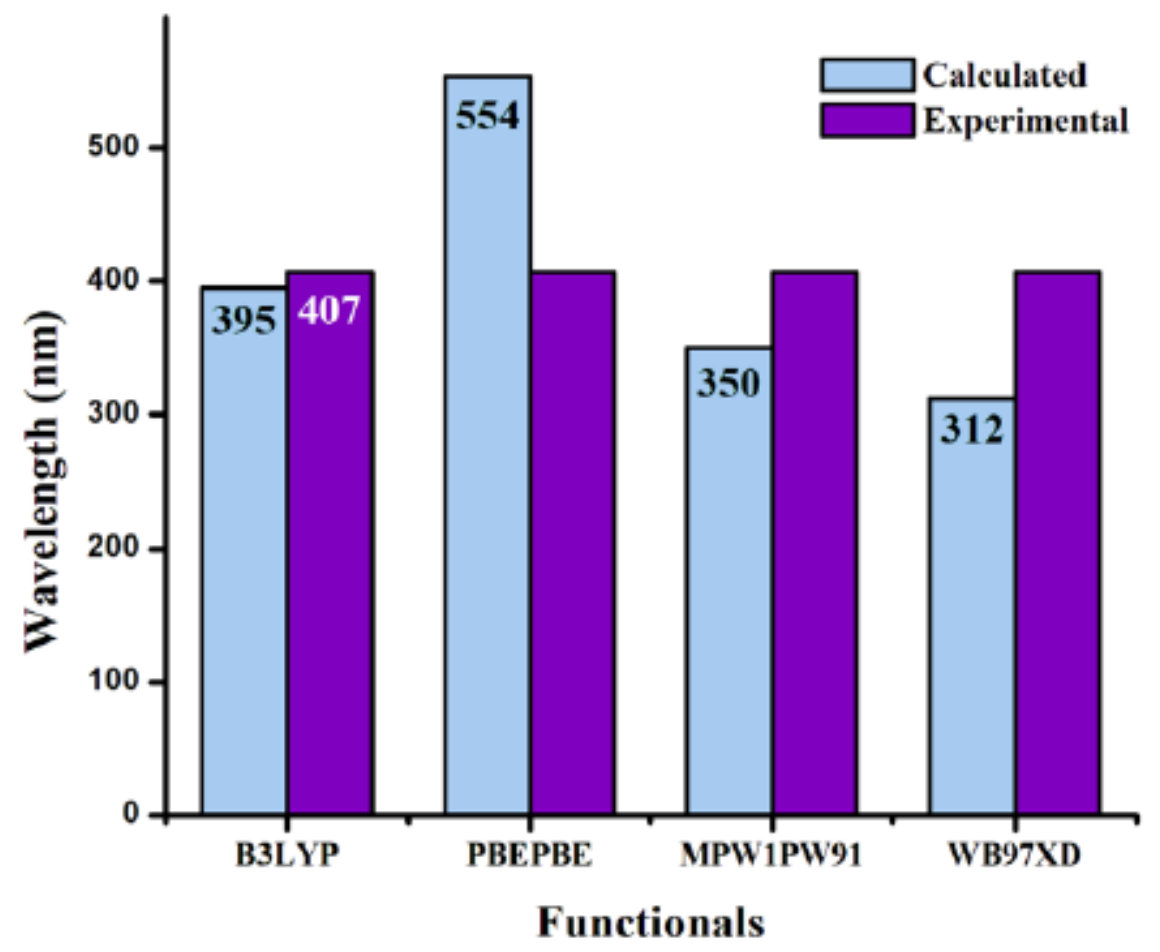

Figure 2

Column chart display demonstrating the calculated and experimental $\lambda$ max at four distinct DFT methods. 


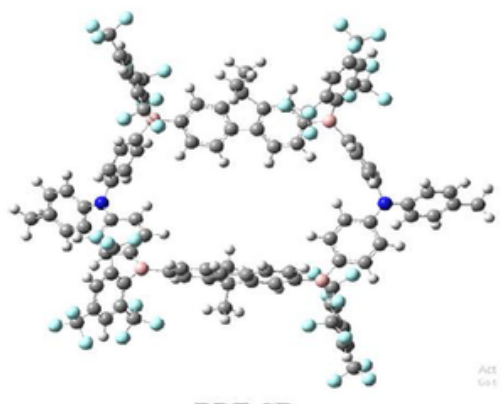

BDT-CR
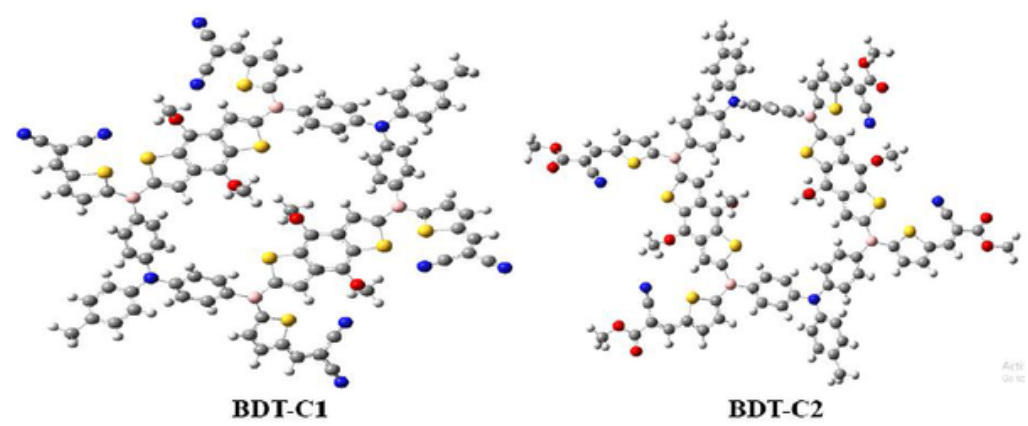

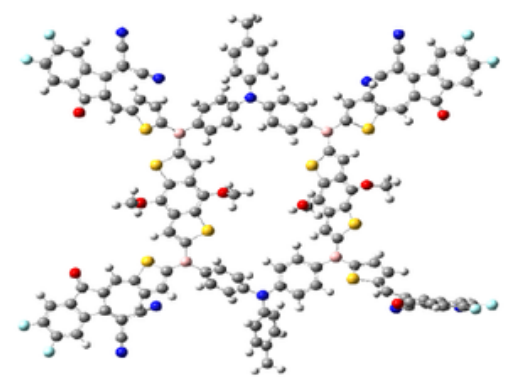

BDT-C3

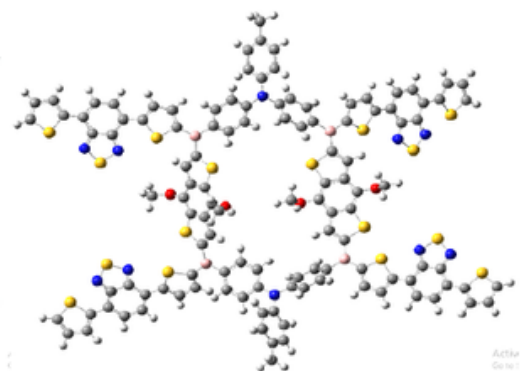

BDT-C4

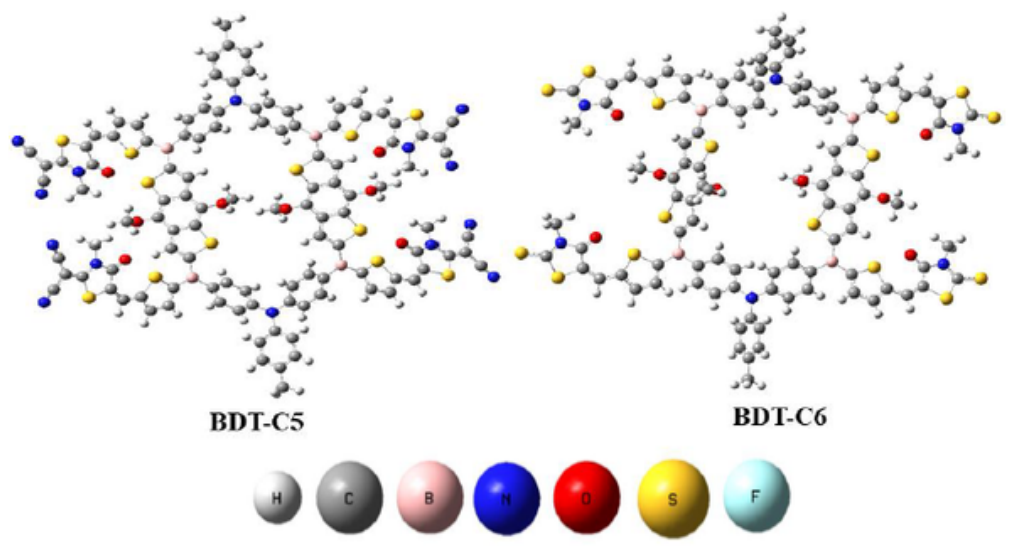

\section{Figure 3}

Optimized geometries of the BDT-CR and designed cross conjugated BDT-C1 to BDT-C6 molecules 

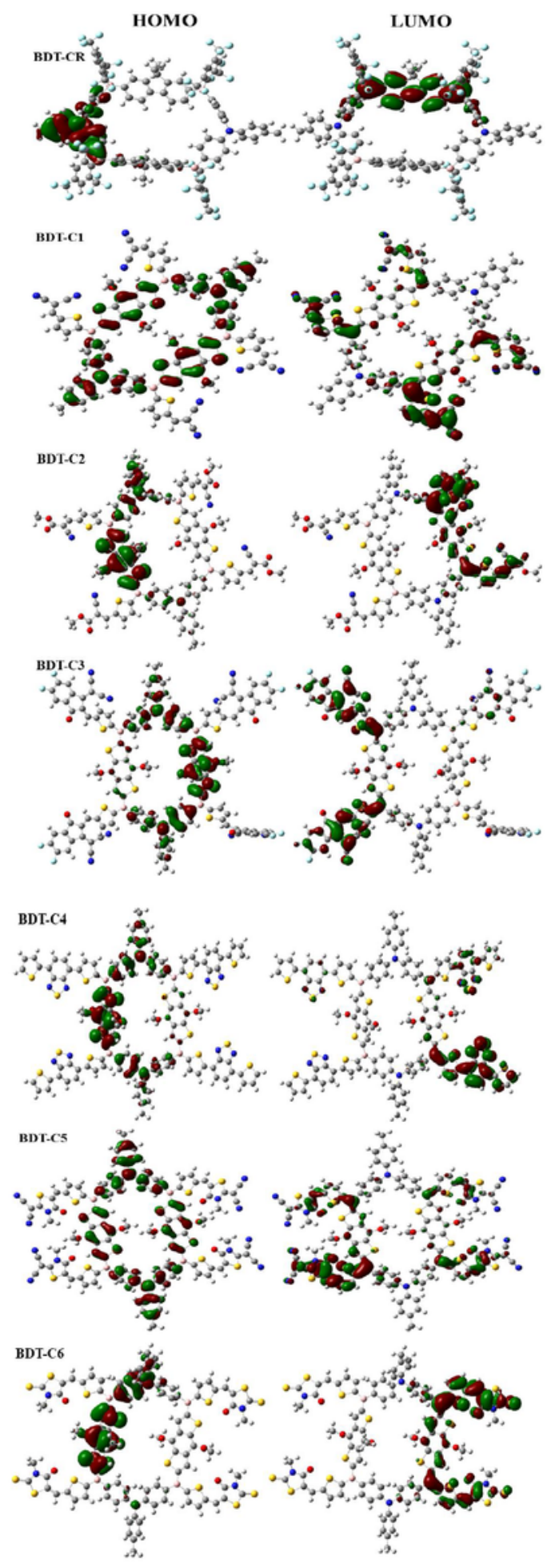

Figure 4

HOMO-LUMO distribution pattern of BDT-CR and cross conjugated BDT-C1 to BDT-C6 molecules. 

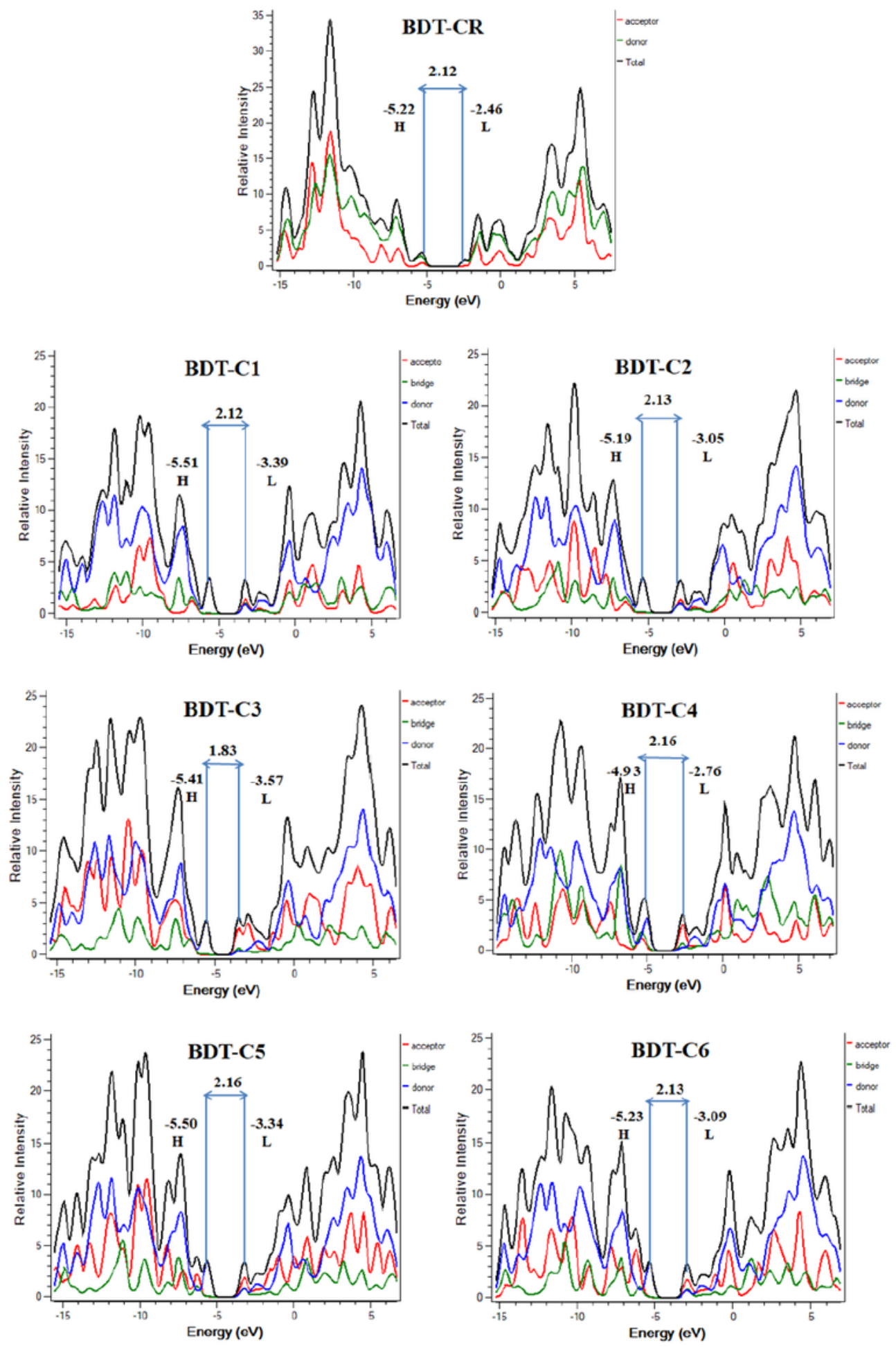

Figure 5

Density of state (DOS) of BDT-CR and designed cross conjugated molecules BDT-C1-BDT-C6 around $\mathrm{H}=\mathrm{HOMO}$ and $\mathrm{L}=\mathrm{LUMO}$ 
(a)

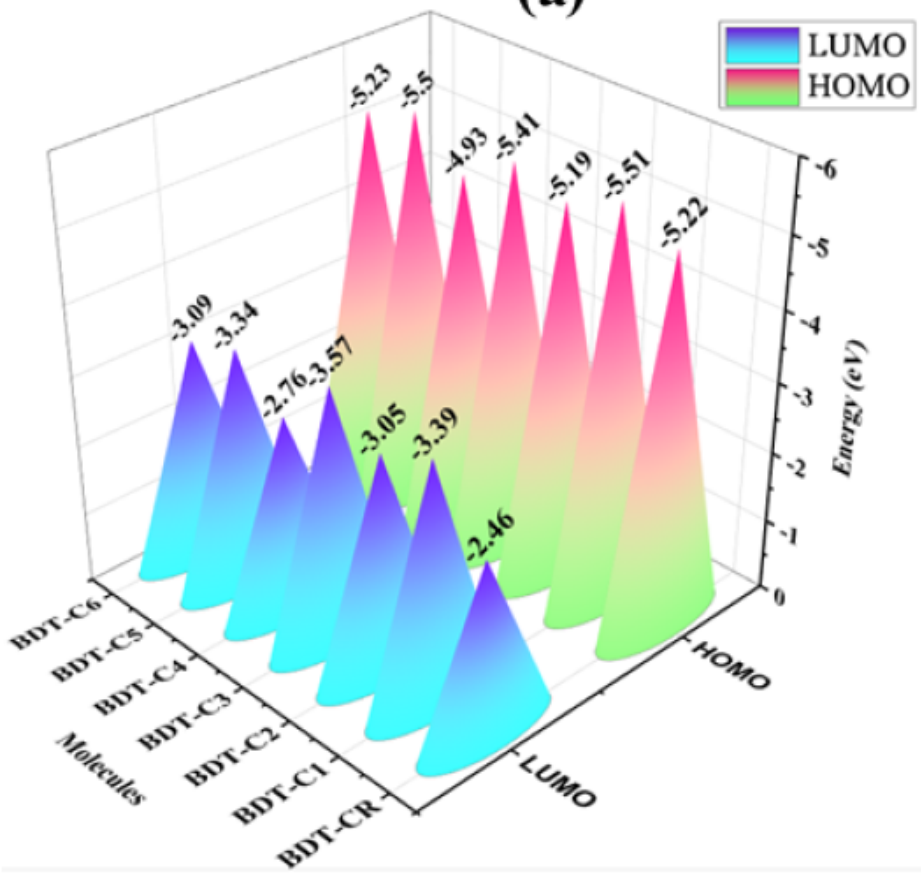

(b)

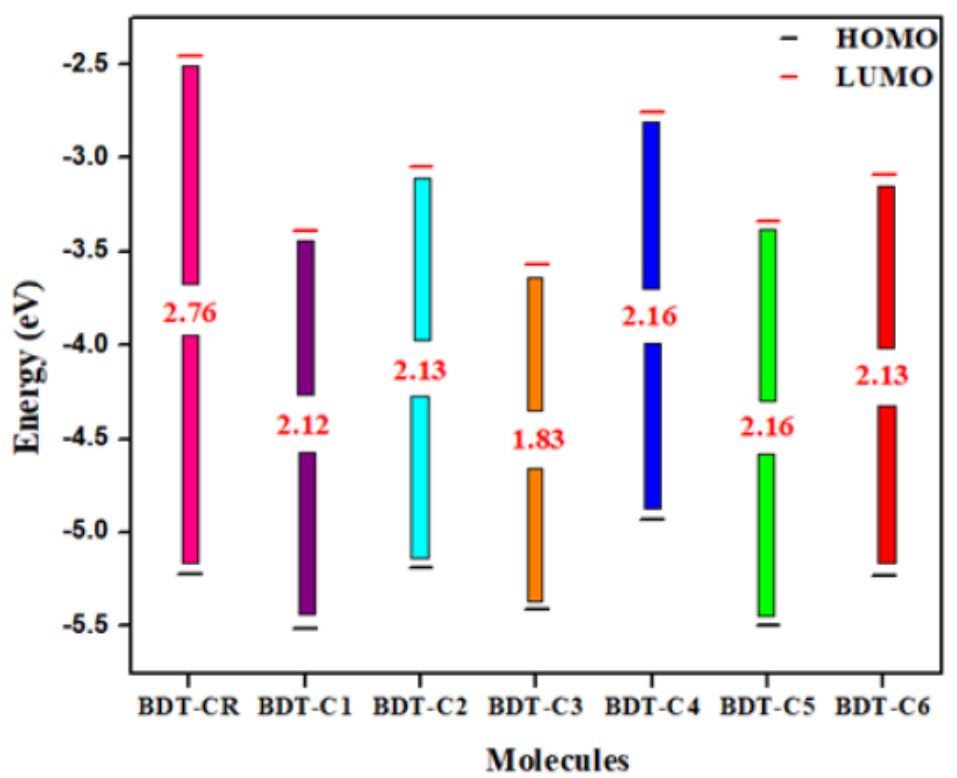

Figure 6

Graphical depiction of (a) HOMO and LUMO energy levels (b) band gap for BDT-CR and BDT-C1 to BDT-C6 molecules.
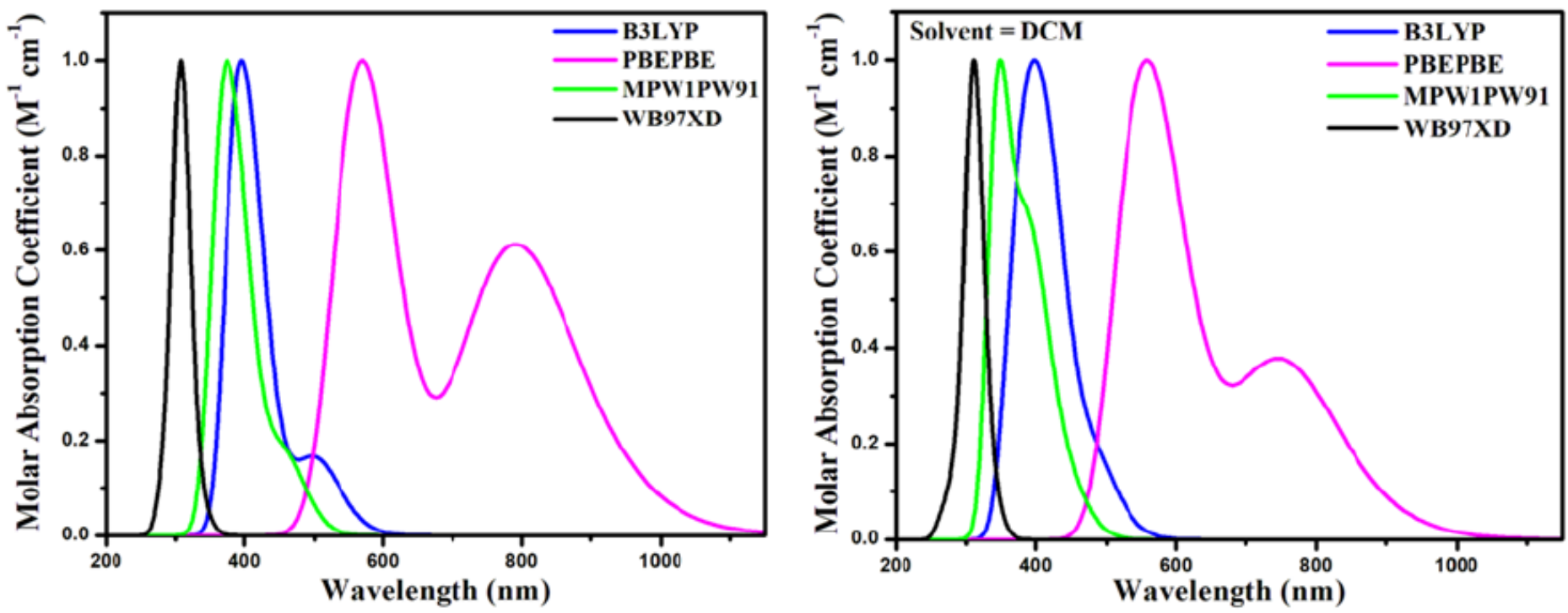

Figure 7

UV-visible absorption spectra for reference BDT-CR with four different functionals in both chloroform (right) and gaseous phases (left). 

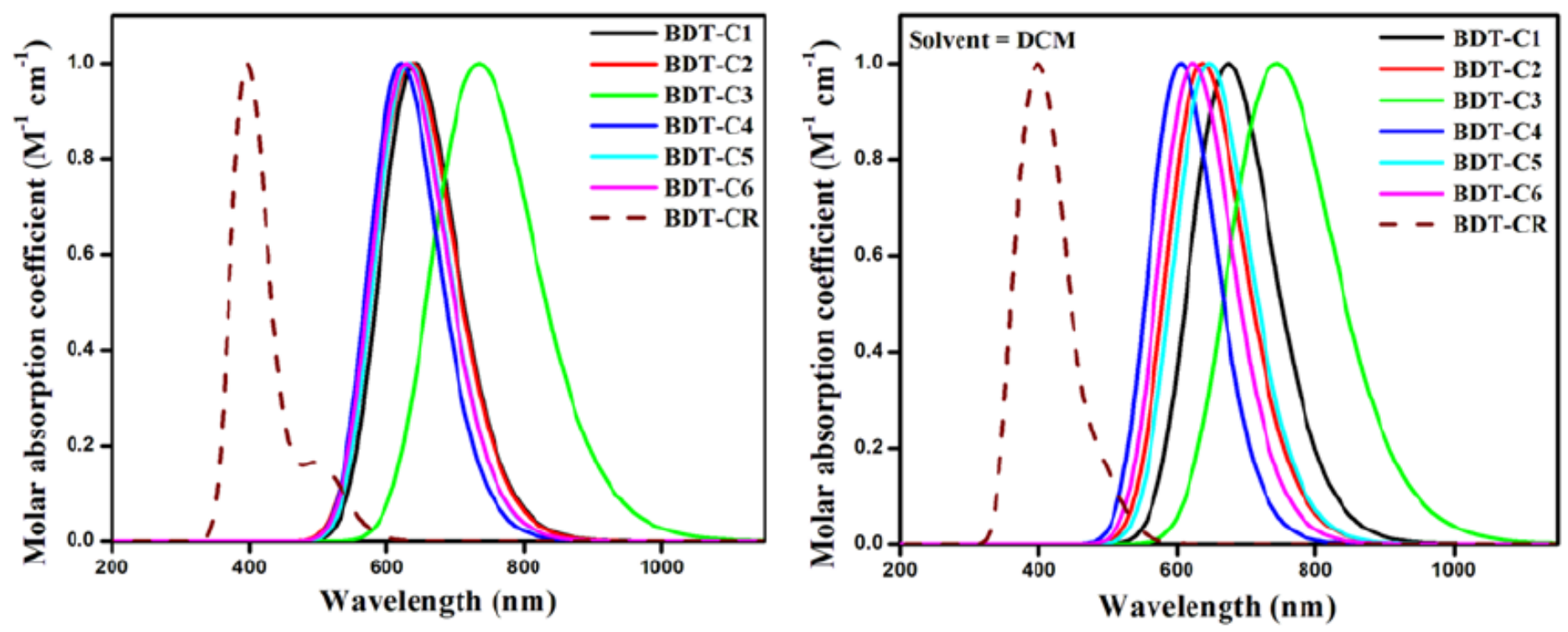

Figure 8

UV-Visible absorption spectra for cross conjugated designed molecules BDT-C1 to BDT-C6 in solvent (right) and gaseous phase (left) 

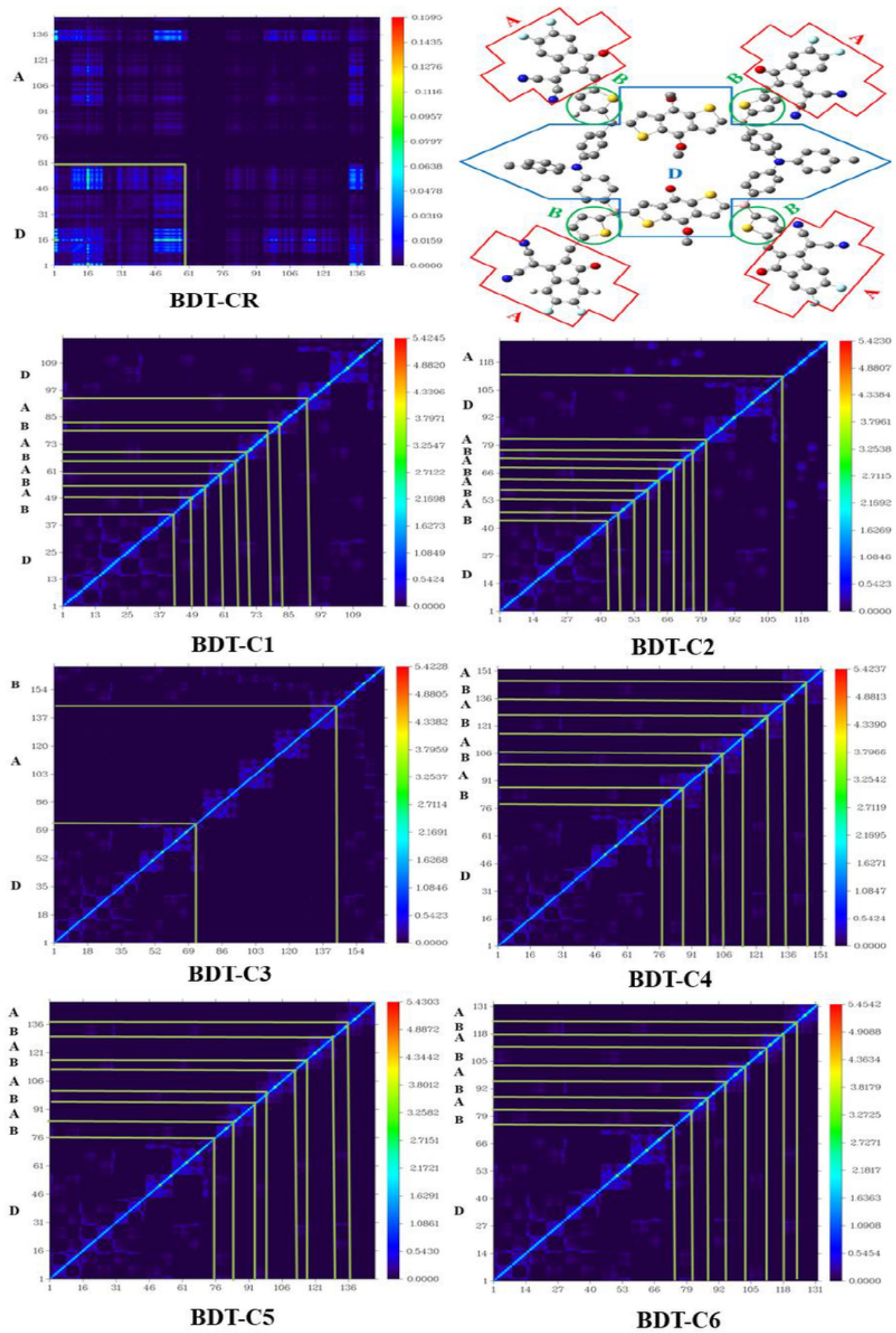

Figure 9

Transition density matrix map for BDT-CR and designed BDT-C1 to BDT-C6 molecules where D = donor, B $=$ bridge and $\mathrm{A}=$ =acceptor. 


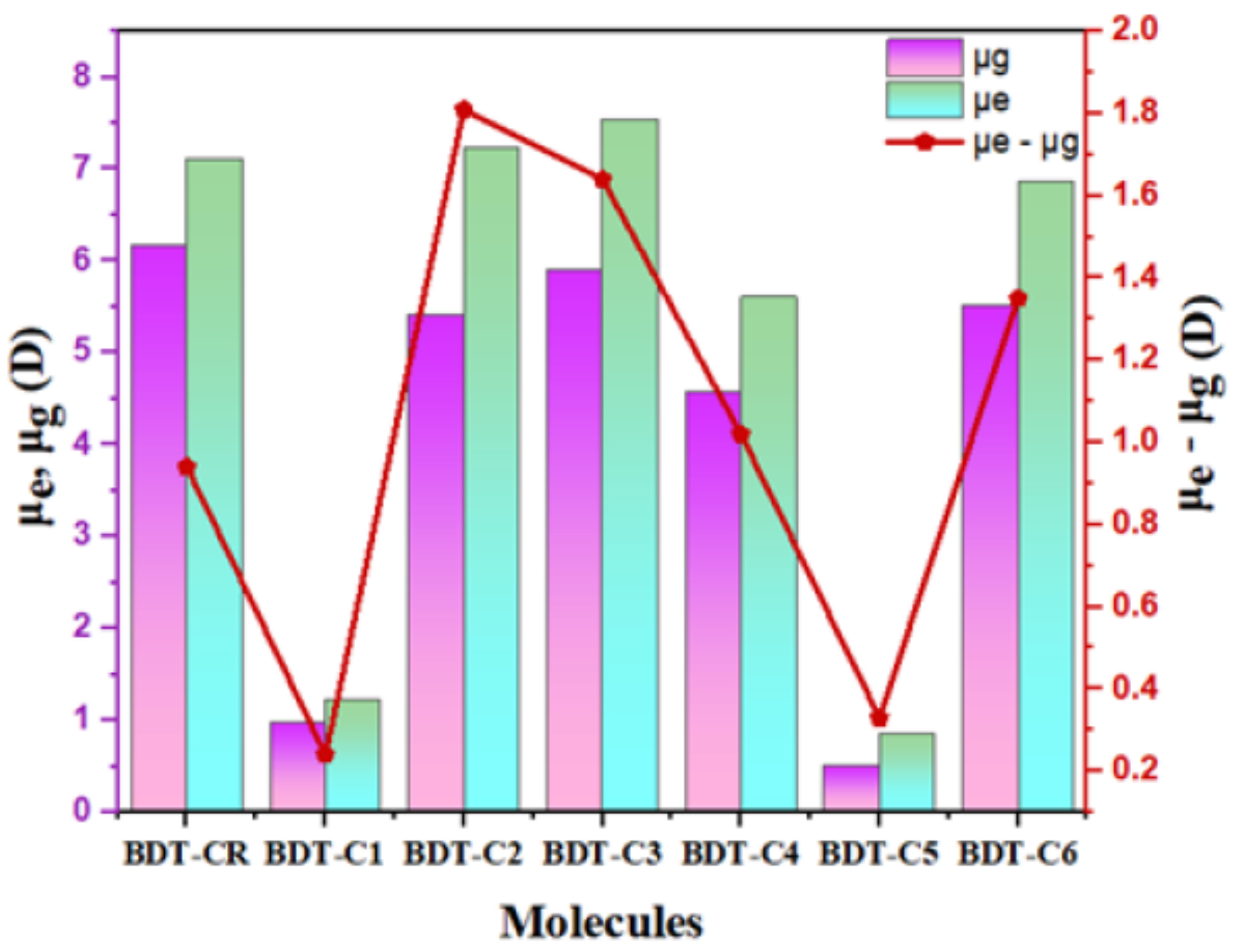

Figure 10

Graphical display of dipol moments for BDT-CR and designed BDT-C1 to BDT-C6 molecules.

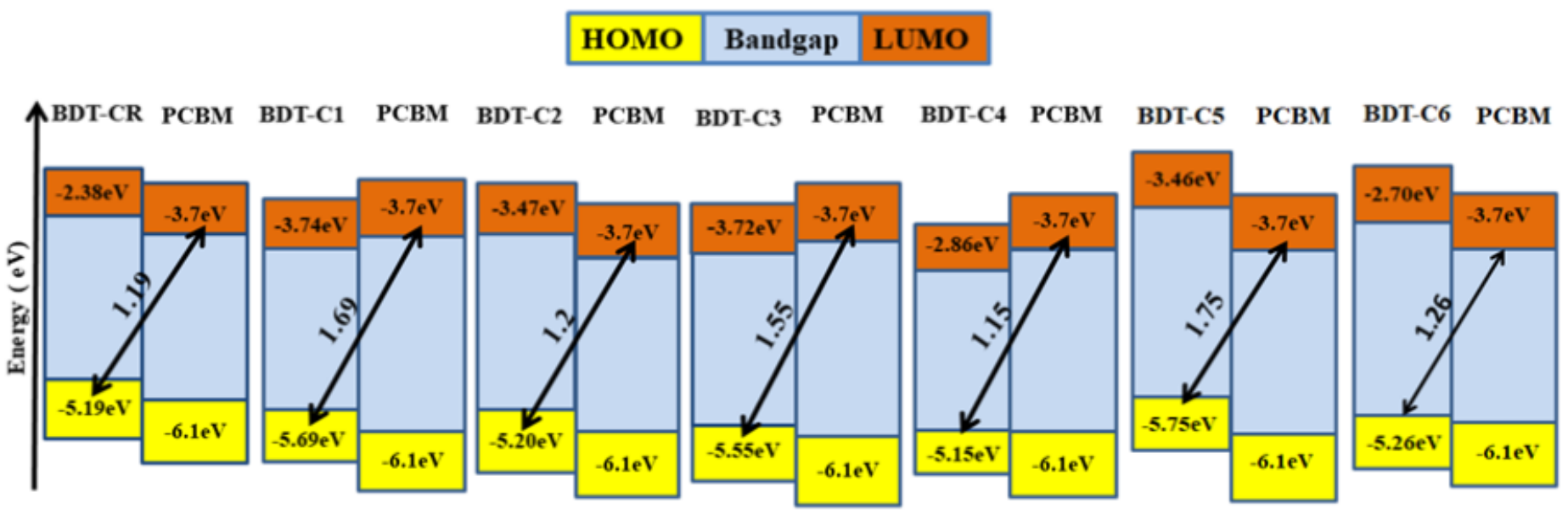

Figure 11

Estimated open circuit voltage of BDT-CR and BDT-C1 to BDT-C6 designed cross conjugated molecules with regard to PCBM polymer acceptor molecule.cross conjugated molecules 


\section{Supplementary Files}

This is a list of supplementary files associated with this preprint. Click to download.

- SUPPORTINGINFORMATION.docX 